\title{
Spring-assisted cranial vault expansion in the setting of multisutural craniosynostosis and anomalous venous drainage: case report
}

\author{
Melinda A. Costa, MD, ${ }^{1}$ Laurie L. Ackerman, MD, ${ }^{2}$ Sunil S. Tholpady, MD, PhD, ${ }^{1}$ \\ S. Travis Greathouse, MD, ${ }^{1}$ Youssef Tahiri, MD, MSc, ${ }^{1}$ and Roberto L. Flores, MD1,3 \\ 1Division of Plastic Surgery and 2Department of Neurosurgery, Riley Hospital for Children, Indiana University School of Medicine, \\ Indianapolis, Indiana; and ${ }^{3}$ Department of Plastic Surgery, NYU Langone Medical Center, New York, New York
}

Patients with multisutural craniosynostosis can develop anomalous venous connections between the intracranial sinuses and cutaneous venous system through enlarged emissary veins. Cranial vault remodeling in this subset of patients carries the risk of massive intraoperative blood loss and/or occlusion of collateral draining veins leading to intracranial venous hypertension and raised intracranial pressure, increasing the morbidity of cranial expansion. The authors report the use of spring-mediated expansion as a technique for cranial reconstruction in which the collateral intracranial venous drainage system can be preserved.

A patient with bilateral lambdoid, sagittal, and unicoronal synostosis presented for cranial reconstruction. A tracheostomy and ventriculoperitoneal shunt were placed prior to intervention. At the time of reconstruction, a Luckenschadel skull abnormality and Chiari malformation Type I were present. A preoperative CT venogram demonstrated large collateral superficial occipital veins, small bilateral internal jugular veins, and hypoplastic jugular foramina. Collateral flow from the transverse and sigmoid sinuses through large occipital emissary veins was seen. Spring-mediated cranial vault expansion was performed with care to preserve the large collateral veins at the occipital midline. Four springs were placed at each lambdoid and the posterior and anterior sagittal sutures following 1-cm strip suturectomies. Removal of the springs was performed 2 months postoperatively.

Cranial vault expansion was performed without disturbing the aberrant intracranial/extracranial venous collateral system. Estimated blood loss was $150 \mathrm{ml}$. A CT scan obtained 3 months postoperatively showed resolution of the Luckenschadel deformity and a $40 \%$ volumetric increase in the skull compared with the preoperative CT.

Patients with anomalous venous drainage patterns and multisutural synostosis can undergo spring-mediated cranial vault expansion while preserving the major emissary veins draining the intracranial sinuses. Risks of blood loss, intracranial venous hypertension, and increased intracranial pressure may be decreased compared with traditional techniques of repair.

http://thejns.org/doi/abs/10.3171/2014.12.PEDS14604

KEY WORDS craniosynostosis; anomalous venous drainage; cranial expansion; spring-assisted cranioplasty; craniofacial

$\mathrm{P}$ ATIENTS with complex, or multisutural, craniosynostosis are at the highest risk of developing increased intracranial pressure (ICP) unless cranial vault expansion is performed. Unfortunately, it is this same patient population that is at most risk of developing anomalous intracranial venous drainage patterns. . $2,4,10,12,14,19,20,22,23$ These intracranial-to-extracranial collateral vessels develop in the occipital region, hindering safe exposure of the poste- rior calvaria during posterior vault remodeling and Chiari decompression. There are multiple reports in which cranial vault remodeling and Chiari decompression repairs were abandoned due to anomalous venous drainage patterns, either identified through preoperative imaging or intraoperatively. ${ }^{1,2,8,23}$ The precedent for this practice was set by the case of a patient who died after posterior vault remodeling and in whom anomalous venous drainage

ABBREVIATIONS BLSS = bilateral lambdoid and sagittal synostosis; ICP = intracranial pressure; VP = ventriculoperitoneal.

SUBMITTED October 31, 2014. ACCEPTED December 19, 2014

INCLUDE WHEN CITING Published online April 10, 2015; DOI: 10.3171/2014.12.PEDS14604.

DISCLOSURE The authors report no conflict of interest concerning the materials or methods used in this study or the findings specified in this paper. 
patterns were identified postoperatively. ${ }^{26,27}$ Subsequent reports have regarded the presence of anomalous intracranial venous drainage as a contraindication to posterior vault remodeling or other posterior fossa procedures. ${ }^{1,2,8}$ As a result, traditional cranial vault expansion techniques may have limited application in the setting of anomalous venous drainage patterns.

Spring-mediated cranial expansion was introduced by Lauritzen et al. in $1998^{13}$ and has since been effectively applied to infants with single- and multisutural craniosynostosis. ${ }^{3,7,8,28}$ As the operative time is relatively brief and the degree of surgical dissection is limited, spring-mediated cranial expansion has been purported as a safe alternative technique for cranial vault expansion. We present a patient with bilateral lambdoid, sagittal, and unicoronal craniosynostosis, Chiari malformation Type I, and hydrocephalus in whom spring-mediated posterior remodeling was performed in the setting of anomalous intracranial venous drainage.

\section{Case Report}

\section{History and Examination}

Institutional review board approval was obtained for this study. A complex neonate was transferred to our tertiary care children's hospital for respiratory failure after being born at 28 weeks due to premature rupture of membranes. Physical examination was notable for severe turribrachycephaly, midface hypoplasia, low-set ears, and clubbed feet. Family history was negative for similar anomalies. A genetic evaluation revealed an Xp21.1 deletion. Radiographic imaging included a skull radiograph demonstrating brachycephaly and thumb printing (Fig. 1). A CT scan demonstrated multisutural synostosis involv-

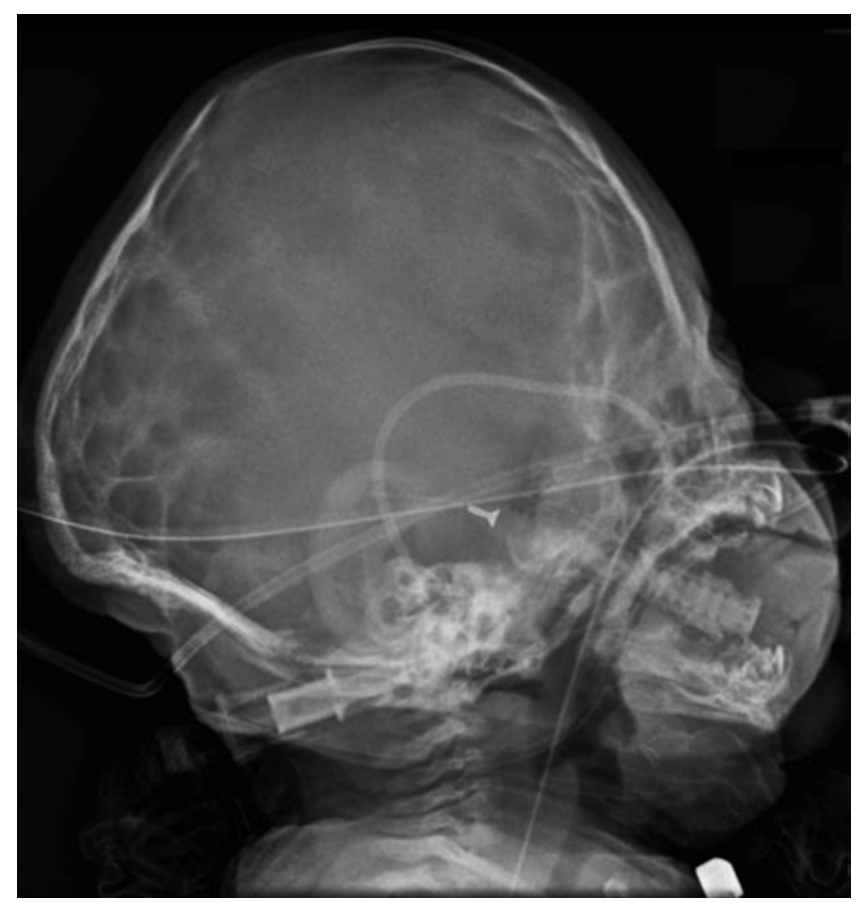

FIG. 1. Preoperative lateral radiograph obtained at 9 weeks of age. Luckenschadel abnormality and brachycephaly are present. ing the bilateral lambdoid, posterior sagittal, and medial aspects of the right coronal suture, Luckenschadel cranial abnormality, and occipital concavity in the midline (Fig. $2)$. The phenotype resembled bilateral lambdoid and sagittal synostosis (BLSS), first reported in 1976. ${ }^{15,16}$ The hallmark pattern of bilateral lambdoid and posterior sagittal suture fusion gave rise to the term "Mercedes Benz" syndrome..$^{15}$ This heterogeneous disorder, recently described in a series of 11 patients, results in a characteristic head shape with frontal bossing, turribrachycephaly, biparietal narrowing, occipital concavity, and inferior displacement of the ears. ${ }^{11}$ Three patients in that series had partial/complete synostosis of a single coronal suture, similar to our patient. MRI revealed a Chiari I malformation with $1-\mathrm{cm}$ cerebellar tonsillar herniation, mild enlargement of the lateral and third ventricles, a compressed fourth ventricle, small posterior fossa, and decreased gyration of the occipital/temporal lobes.

The patient underwent resection of a mediastinal hemangioma at the time of infancy followed by a tracheostomy at 3 months of age to treat mixed central and obstructive sleep apnea associated with pharyngomalacia and possibly the Chiari I malformation. At 6 months of age, a repeat CT scan showed worsened Chiari I malformation with 2 $\mathrm{cm}$ of cerebellar tonsillar herniation and increased hydrocephalus; a ventriculoperitoneal (VP) shunt was placed. The VP shunt was placed in an anterior location, at the right frontal region, in anticipation of a posterior cranial reconstruction. A preoperative CT venogram obtained at 6 months of age showed large collateral superficial occipital veins, small bilateral internal jugular veins, and hypoplastic jugular foramina. Collateral flow from the transverse and sigmoid sinuses through large occipital emissary veins was seen (Fig. 3). The patient's treatment plan was delayed due to an episode of tracheitis.

\section{Operation}

The operation was performed when the patient was 9 months old. Given the need for expansion in multiple vectors, inconsistent calvarial thickness, and the desire to preserve the venous collateral system of the scalp, spring-mediated posterior expansion was planned. A 3D rendering was used to assist in planning the osteotomies and spring location (Fig. 4). Spring-mediated cranial vault expansion was performed using a directed bicoronal exposure of the
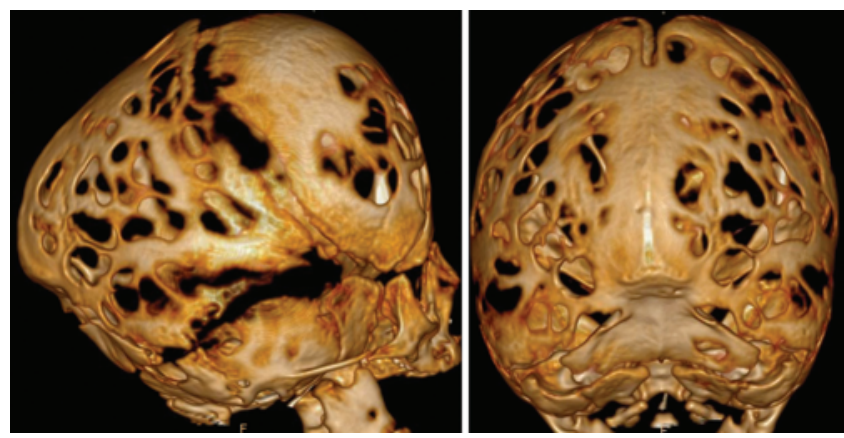

FIG. 2. Preoperative 3D CT scans illustrating multiple irregular cranial defects. Left: Lateral view. Occipital flattening and turribrachycephaly are seen. Right: Posterior view demonstrating bilateral lambdoid, sagittal, and unicoronal synostosis. Figure is available in color online only. 


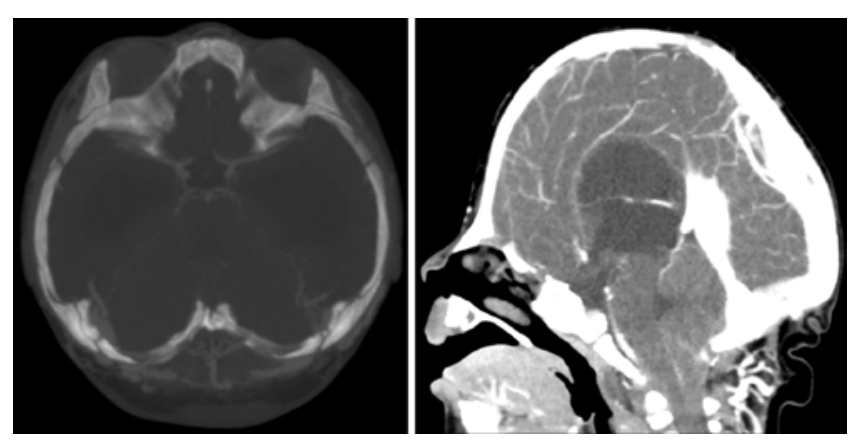

FIG. 3. Axial (left) and sagittal (right) CT scans illustrating the confluence of veins in the occipital region and transosseous venous drainage through large occipital emissary veins.

cranial sutures. During exposure, the veins communicating with the scalp were noted as a whole to be larger than is typical for patients with single- or bisutural craniosynostosis of a similar age. Care was taken not to disturb the large, aberrant collateral veins at the occipital midline concavity and major cranial emissary veins by avoiding dissection in this area. A strip craniectomy was performed on the bilateral lambdoid and coronal sutures as well as the sagittal suture. Four cranial springs (Osteomed) were placed: 1 at each lambdoid suture and 2 at the sagittal suture along the posterior and anterior aspects following 1-cm strip suturectomies (Fig. 5). A 7-N force was applied to each spring and an immediate cranial vault expansion was appreciable at the end of the procedure. Estimated blood loss was $150 \mathrm{ml}$.

\section{Postoperative Course}

The patient's head was kept in the lateral position to avoid pressure injury to the scalp. There was no occurrence of surgical site infection or spring exposure throughout the postoperative course. Spring removal was performed 2 months postoperatively; there were no post-

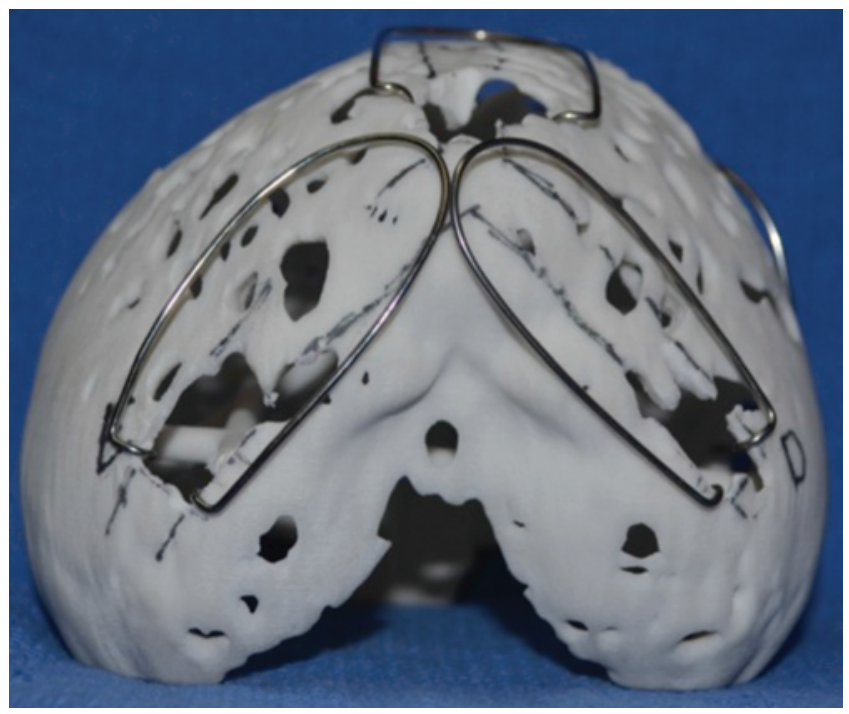

FIG. 4. Three-dimensional rendering of proposed sites of suturectomies and spring placement at bilateral lambdoid and sagittal sutures, posterior view. Figure is available in color online only.

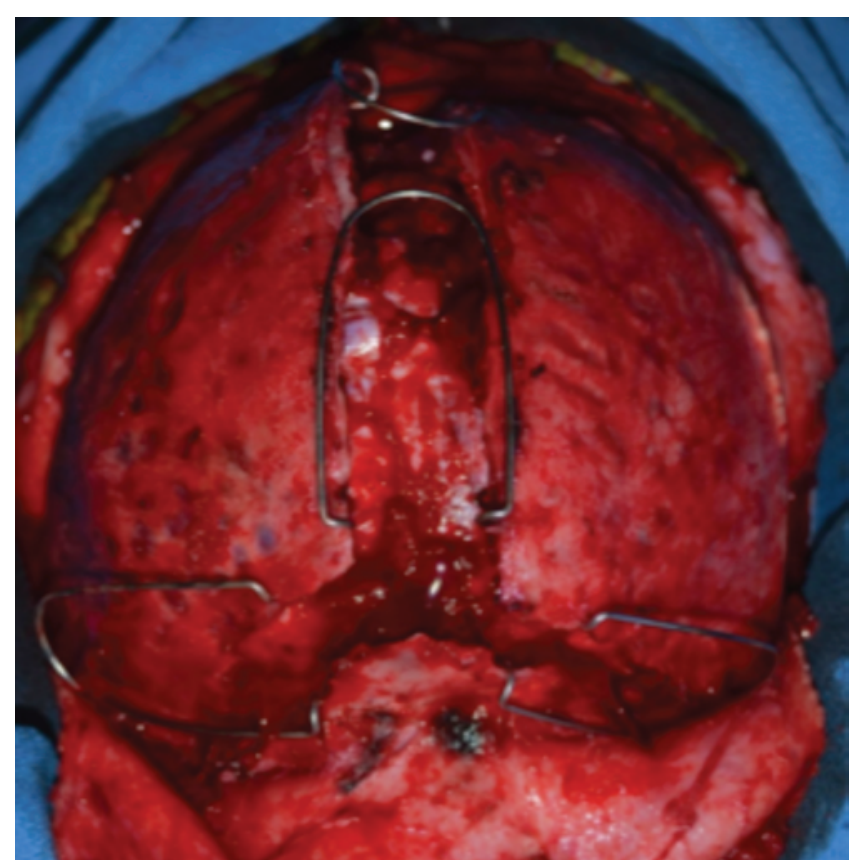

FIG. 5. Intraoperative view of the osteotomies and spring placement. Reflection of the scalp in the occipital midline was avoided to preserve large extracranial veins contributing to intracranial venous drainage. Figure is available in color online only.

operative complications. A CT scan obtained 3 months postoperatively showed resolution of the Luckenschadel abnormality and a $40 \%$ volumetric increase in the skull compared with the preoperative CT (Fig. 6). The Chiari malformation was unchanged, and the degree of hydrocephalus was improved compared with preoperative imaging. Of note, the postoperative CT scan demonstrated a portion of the left frontal bone overriding at the location of the osteotomy. We anticipate that this irregularity will improve with brain growth and subsequent calvarial remodeling. Adequate follow-up will be needed to determine the degree of resolution. Over the 5-month follow-up period, the patient's developmental status improved based on developmental milestones.
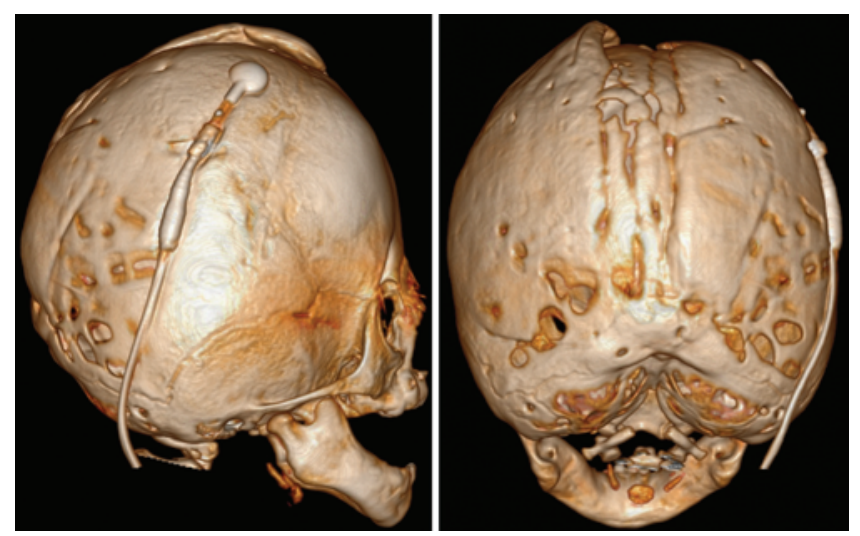

FIG. 6. Lateral (left) and posterior (right) postoperative 3D CT scans obtained 3 months after spring placement, demonstrating improvement in the Luckenschadel abnormality and skull shape. Figure is available in color online only. 


\section{Discussion}

Several studies have reported an association between anomalous intracranial venous drainage patterns and complex craniosynostosis. Jeevan et al. identified abnormal transosseous venous drainage patterns on CT venography in 9 of 11 patients with syndromic craniosynostosis. ${ }^{12}$ Booth et al. compared children with and without craniosynostosis, noting a statistically significant, $23 \%$ decrease of the jugular foramen on the right side in patients with craniosynostosis. ${ }^{4}$ No statistically significant difference was found on the left. Earlier studies identified associations between venous hypertension and hydrocephalus, ${ }^{17,21,24}$ and many have proposed that venous hypertension may lead to raised ICP. ${ }^{10,19,25}$ Taylor et al. evaluated venous drainage patterns among 23 patients with multisutural craniosynostosis who ranged in age from 3 months to 8 years; the majority were younger than 3 years of age. ${ }^{25}$ Eighteen digital subtraction angiograms showed greater than $50 \%$ stenosis at the sigmoid jugular sinus complex on one (7 patients) or both (11 patients) sides; transosseous venous drainage was also identified. Eleven of these patients showed florid collateral circulation in the stylomastoid emissary vein region. All but 2 patients had raised ICP and none had hydrocephalus, suggesting that impaired venous drainage led to intracranial venous hypertension and raised ICP. MartinezPerez et al. described the occurrence of a postoperative arteriovenous fistula in the mastoid region in a 4-year-old patient with Crouzon syndrome following posterior calvarial vault remodeling. ${ }^{14}$ The authors suggested that intracranial venous hypertension resulted from impaired drainage due to stenosis of the jugular foramina, leading to scalp vein dilation and subsequent traumatic arteriovenous fistula.

There are case reports and case series describing problems with occipital procedures in the setting of abnormal intracranial venous drainage. As a result, several authors have come to recommend against intervention if anomalous drainage patterns are present. The most notable case was reported by Thompson et al.: a 9-year-old patient with Kleeblattschädel deformity in whom ligation of communicating scalp veins during reflection of the scalp led to sudden intraoperative intracranial hypertension, abandonment of the operation, intractable intracranial hypertension, and subsequent death. ${ }^{26,27}$ The authors later described a patient with left lambdoid partial sagittal synostosis for whom posterior vault remodeling was abandoned intraoperatively due to an unexpected venous hum in the occipital region. ${ }^{2}$ MRI and carotid angiography revealed hypoplastic internal jugular veins and transosseous venous drainage via emissaries to the external jugular and vertebral systems. The anomalous venous drainage pattern was regarded as a contraindication to vault expansion. Al-Otibi et al. reported anomalous venous anatomy in a patient with Crouzon syndrome and Chiari malformation with syrinx in which decompression was avoided due to anomalous venous anatomy. ${ }^{1}$ These authors recommended that CT venography be performed preoperatively in all patients with multisutural craniosynostosis. Sandberg et al. reported on 2 patients with multisutural craniosynostosis, Chiari I malformation, and prior VP shunt placement in whom posterior fossa decompression was avoided due to anomalous venous drainage patterns. ${ }^{23}$ Preoperative CT angiography demonstrated hypoplastic jugular foramina and abnormal transosseous venous drainage to the neck and retromastoid region. In addition, Sanchez et al. described the onset of facial edema 21 months after correction of BLSS at 2 months of age via strip craniectomy and spring placement; bilateral jugular foraminal stenosis was subsequently identified..$^{22}$

In this report, posterior vault remodeling was safely accomplished by preserving the intracranial/extracranial venous collateral system. CT venography was performed preoperatively to identify the venous anatomy. Hypoplastic jugular foramina were seen, as were large emissary veins draining into scalp veins in the occipital midline. During the operation, disruption of the venous system in this area was avoided by performing a scalp exposure limited to the cranial sutures and avoiding the central occiput, thereby limiting blood loss and risk of developing intracranial venous hypertension. A strip craniectomy was then performed, followed by a spring-mediated cranial vault expansion. We conclude that the presence of anomalous venous drainage patterns in patients with multisutural craniosynostosis does not necessarily represent a contraindication to cranial vault expansion. Spring-assisted posterior vault expansion has been reported for multisutural as well as for single-suture craniosynostosis. ${ }^{3,7,28}$ De Jong et al. described a series of 15 expansions in patients with multisutural synostosis; the presence of anomalous venous drainage, however, is regarded as a contraindication to care. ${ }^{8}$

In addition to venous drainage anomalies, our patient presented with a Chiari I malformation that remained unchanged after cranial reconstruction. While the association between Chiari malformation and craniosynostosis has been reported, $5,6,9,18$ the pathophysiology is unclear. It has been suggested to consist of multiple factors, including cephalocranial disproportion relating to a small posterior fossa relative to hindbrain growth, jugular foraminal stenosis, and impaired intracranial venous drainage that may lead to hydrocephalus and/or intracranial hypertension. ${ }^{6,18}$ Premature lambdoid suture closure has been proposed to be a cause of cephalocranial disproportion leading to Chiari malformation; it has also been found to be significantly associated with chronic tonsillar herniation in patients with Crouzon syndrome. ${ }^{5}$ Given the multifactorial pathophysiology, posterior vault remodeling may or may not affect an existing Chiari malformation. In addition, it is possible that cases of multisutural synostosis involving the lambdoid suture, such as BLSS, rather than all cases of multisutural synostosis, are associated with impaired intracranial venous drainage. In the absence of confirmatory data, we agree that delineation of venous anatomy should be performed prior to posterior vault procedures in all cases of complex craniosynostosis.

Our choice of technique involved extensive exposure of the scalp to allow visualization of the affected coronal, sagittal, and lambdoid sutures. At the same time, we made sure to avoid disrupting areas with significantly enlarged veins. The major collaterals were located medial and inferior to the lambdoid sutures. We exposed only enough to perform a 1-cm suturectomy in this area. The enlarged scalp and emissary veins present over the occipital bone remained untouched. Although some small collaterals 
may have been sacrificed during scalp elevation, they did not appear to be clinically significant. Nevertheless, longterm follow-up is needed. The patient will be monitored for problems such as facial edema, which has been described following posterior vault remodeling. ${ }^{22}$

Alternate approaches include conventional vault remodeling. This would not have decreased the amount of exposure needed but rather may have increased it, due to the need for additional dissection to provide fixation. In addition, due to the anomalous venous collaterals, we were unable to perform a craniectomy in this area and thus could not address the Chiari I malformation. Given the small posterior fossa volume and worsening Chiari I malformation, we sought to maximally expand the occipital region including the posterior fossa. The springs provided a continual force on the calvarial flaps and skin envelope, resulting in a greater expansion than could have been obtained with conventional remodeling. The gradual expansion of the skin envelope permitted more expansion postoperatively while minimizing the risk of wound breakdown that occurs with over-aggressive intraoperative expansion. Another option for cranial vault remodeling is distractor placement. However, this would have required extensive exposure as well and would not have been able to produce expansion in multiple vectors as was needed in our patient.

Although we safely performed cranial expansion in an infant with anomalous intracranial venous drainage, experience with additional patients and long-term follow-up is needed. In addition, the technique for preserving venous collaterals by avoiding the occipital midline may not be applicable to all patients; the technique will be modified according to the location of the major collateral draining vessels for each individual patient.

\section{Conclusions}

Patients with multisutural synostosis may be candidates for posterior vault expansion despite anomalous intracranial venous drainage patterns. Delineation of venous anatomy is recommended prior to posterior vault expansion in this patient population. The identification and preservation of emissary veins responsible for cerebral sinus drainage is critical in avoiding intracranial venous hypertension. Compared with traditional techniques of repair, blood loss and risk of intracranial venous hypertension may be minimized with spring-mediated distraction. A preexisting Chiari malformation may not improve despite posterior vault expansion.

\section{References}

1. Al-Otibi M, Jea A, Kulkarni AV: Detection of important venous collaterals by computed tomography venogram in multisutural synostosis. Case report and review of the literature. J Neurosurg 107 (6 Suppl):508-510, 2007

2. Anderson PJ, Harkness WJ, Taylor W, Jones BM, Hayward RD: Anomalous venous drainage in a case of non-syndromic craniosynostosis. Childs Nerv Syst 13:97-100, 1997

3. Arnaud E, Marchac A, Jeblaoui Y, Renier D, Di Rocco F: Spring-assisted posterior skull expansion without osteotomies. Childs Nerv Syst 28:1545-1549, 2012

4. Booth CD, Figueroa RE, Lehn A, Yu JC: Analysis of the jugular foramen in pediatric patients with craniosynostosis. $\mathbf{J}$ Craniofac Surg 22:285-288, 2011

5. Cinalli G, Renier D, Sebag G, Sainte-Rose C, Arnaud E, Pierre-Kahn A: Chronic tonsillar herniation in Crouzon's and Apert's syndromes: the role of premature synostosis of the lambdoid suture. J Neurosurg 83:575-582, 1995

6. Cinalli G, Spennato P, Sainte-Rose C, Arnaud E, Aliberti F, Brunelle F, et al: Chiari malformation in craniosynostosis. Childs Nerv Syst 21:889-901, 2005

7. Davis C, MacFarlane MR, Wickremesekera A: Occipital expansion without osteotomies in Apert syndrome. Childs Nerv Syst 26:1543-1548, 2010

8. de Jong T, van Veelen ML, Mathijssen IM: Spring-assisted posterior vault expansion in multisuture craniosynostosis. Childs Nerv Syst 29:815-820, 2013

9. Francis PM, Beals S, Rekate HL, Pittman HW, Manwaring K, Reiff J: Chronic tonsillar herniation and Crouzon's syndrome. Pediatr Neurosurg 18:202-206, 1992

10. Hayward R: Venous hypertension and craniosynostosis. Childs Nerv Syst 21:880-888, 2005

11. Hing AV, Click ES, Holder U, Seto ML, Vessey K, Gruss J, et al: Bilateral lambdoid and sagittal synostosis (BLSS): a unique craniosynostosis syndrome or predictable craniofacial phenotype? Am J Med Genet A 149A:1024-1032, 2009

12. Jeevan DS, Anlsow P, Jayamohan J: Abnormal venous drainage in syndromic craniosynostosis and the role of CT venography. Childs Nerv Syst 24:1413-1420, 2008

13. Lauritzen C, Sugawara Y, Kocabalkan O, Olsson R: Spring mediated dynamic craniofacial reshaping. Case report. Scand J Plast Reconstr Surg Hand Surg 32:331-338, 1998

14. Martinez-Perez D, Vander Woude DL, Barnes PD, Scott RM, Mulliken JB: Jugular foraminal stenosis in Crouzon syndrome. Pediatr Neurosurg 25:252-255, 1996

15. Moore MH, Abbott AH, Netherway DJ, Menard R, Hanieh A: Bilambdoid and posterior sagittal synostosis: the Mercedes Benz syndrome. J Craniofac Surg 9:417-422, 1998

16. Neuhäuser G, Kaveggia EG, Opitz JM: Studies of malformation syndromes of man XXXIX: a craniosynostosis-craniofacial dysostosis syndrome with mental retardation and other malformations: "craniofacial dyssynostosis." Eur J Pediatr 123:15-28, 1976

17. Pierre-Kahn A, Hirsch JF, Renier D, Metzger J, Maroteaux P: Hydrocephalus and achondroplasia. A study of 25 observations. Childs Brain 7:205-219, 1980

18. Ranger A, Al-Hayek A, Matic D: Chiari type 1 malformation in an infant with type 2 Pfeiffer syndrome: further evidence of acquired pathogenesis. J Craniofac Surg 21:427-431, 2010

19. Rich PM, Cox TC, Hayward RD: The jugular foramen in complex and syndromic craniosynostosis and its relationship to raised intracranial pressure. AJNR Am J Neuroradiol 24:45-51, 2003

20. Rollins N, Booth T, Shapiro K: MR venography in children with complex craniosynostosis. Pediatr Neurosurg 32:308315,2000

21. Sainte-Rose C, LaCombe J, Pierre-Kahn A, Renier D, Hirsch JF: Intracranial venous sinus hypertension: cause or consequence of hydrocephalus in infants? J Neurosurg 60:727736,1984

22. Sanchez R, Ward K, St Hilaire H, Mussell J: Basicranial venous anomalies associated with complex nonsyndromic craniosynostosis in a child. J Craniofac Surg 24:1970-1973, 2013

23. Sandberg DI, Navarro R, Blanch J, Ragheb J: Anomalous venous drainage preventing safe posterior fossa decompression in patients with Chiari malformation type I and multisutural craniosynostosis. Report of two cases and review of the literature. J Neurosurg 106 (6 Suppl):490-494, 2007

24. Steinbok P, Hall J, Flodmark O: Hydrocephalus in achondro- 
plasia: the possible role of intracranial venous hypertension. J Neurosurg 71:42-48, 1989

25. Taylor WJ, Hayward RD, Lasjaunias P, Britto JA, Thompson DN, Jones BM, et al: Enigma of raised intracranial pressure in patients with complex craniosynostosis: the role of abnormal intracranial venous drainage. J Neurosurg 94:377-385, 2001

26. Thompson D, Hayward R, Jones B: Anomalous intracranial venous drainage: implications for cranial exposure in the craniofacial patient. Plast Reconstr Surg 95:1126, 1995

27. Thompson DN, Hayward RD, Harkness WJ, Bingham RM, Jones BM: Lessons from a case of kleeblattschädel. Case report. J Neurosurg 82:1071-1074, 1995

28. Tunçbilek G, Kaykçoğlu A, Bozkurt G, Akalan N: Springmediated cranioplasty in patients with multiple-suture syn- ostosis and cloverleaf skull deformity. J Craniofac Surg 23:374-377, 2012

\section{Author Contributions}

Conception and design: Flores, Ackerman. Acquisition of data: Costa, Greathouse. Analysis and interpretation of data: Flores, Costa. Drafting the article: Costa. Critically revising the article: Flores, Ackerman, Tholpady, Tahiri.

\section{Correspondence}

Roberto L. Flores, Department of Plastic Surgery, NYU Langone Medical Center, 307 E. 33rd St., Lower Level, New York, NY 10016. email: roberto.flores2@nyumc.org. 